\title{
The role of calprotectin in rheumatoid arthritis
}

\author{
Qin Wang, Weiqian Chen, Jin Lin \\ Division of Rheumatology, First Affiliated Hospital, \\ College of Medicine, Zhejiang University, Hangzhou, Zhejiang Province, China
}

\section{ABSTRACT}

Rheumatoid arthritis (RA) is an inflammatory autoimmune disease characterized by inflammatory cell infiltration, high levels of cytokines, and erosion of cartilage and bone in joints. Calprotectin (CLP), as a recently described member of $\mathrm{S} 100$ family proteins, is a heterodimeric complex of S100A8 and S100A9. Currently, plenty of studies have indicated significantly increased serum and synovial fluid levels of CLP in patients with RA. It was reported that CLP was related to cell differentiation, migration, apoptosis, and production of pro-inflammatory factors in RA. In addition, there are the positive relationships between serum, synovial CLP and traditional acute phase reactants, disease activity, ultrasound and radiographic progression of joints, and treatment response of RA. In this review, we mainly discuss the role of CLP in the pathogenesis of RA as well as its potential to estimate clinical disease progression of RA patients.

Key words: rheumatoid arthritis, calprotectin, inflammation

\section{INTRODUCTION}

Rheumatoid arthritis (RA) is a progressive autoimmune disease characterized as elevated inflammatory cytokines, excessive synovial hyperplasia, and erosion of cartilage and bone in the articular cavity. However, the exact pathogenesis of RA is still unclear. Calprotectin (CLP), an S100A8/S100A9 heterodimer, is a member of the S100 family proteins. Recent studies have observed an increased CLP level in blood and synovial fluid (SF) from RA patients. The levels of CLP were associated with RA disease activity index such as C-reactive protein (CRP) and erythrocyte sedimentation rate (ESR). CLP levels are also correlated with ultrasounddetermined synovitis, joint radiographic progression and the therapeutic reaction of RA patients. ${ }^{[1-7]}$ CLP performs various biological functions via interaction with Toll-like receptor 4 (TLR4) in RA. ${ }^{[8]}$ Its functions are largely divided into two parts: intracellular and extracellular effects. CLP involves in cytoskeleton regulation and cell migration. ${ }^{[9]}$ When released, it mediates cell differentiation, activation, migration and apoptosis, and promotes the production of pro-inflammatory mediator. ${ }^{[10,11]}$ Accumulating evidence has suggested CLP may exert arresting functions in the pathogenesis of RA. Therefore, CLP may be recommended as a potential biomarker in RA. In this review, we aim to summarize the signaling pathway of CLP in the pathomechanism and disease progression of RA patients.

\section{S100 PROTEINS, CLP AND TLR4}

\section{S100 proteins and CLP}

Moore $^{[12]}$ first isolated S100 proteins from bovine brain tissue in 1965, and the name 'S100' originated from its solubility in a saturated ammonium sulfate solution. Up to now, more than twenty S100 family members are confirmed, including S100A8, S100A9, S100A1, S100A12, S100B. S100 is a superfamily of calcium-binding proteins exhibiting intracellular and extracellular functions. ${ }^{[13]}$ They seize target molecules (such as enzymes, cytoskeletal proteins) and participate in cell motility and $\mathrm{Ca}^{2+}$ homeostasis within cells. S100 proteins are 
actively or passively secreted into the extracellular space in case of cell activation or necrosis. ${ }^{[14]}$ The extracellular proteins, identified as damage-associated molecular patterns (DAMPs), become danger signals and are associated with cardiac disease, infectious disease, nervous system disease, cancer and autoimmune disease. ${ }^{[8,14-16]}$

CLP (also known as myeloid-related proteins MRP8/ MRP14, calgranulin A and B, and L1 protein) is a heterodimeric complex of two EF-hand calcium-binding proteins, S100A8 and S100A9, which are both encoded by highly conserved genes clustered on chromosome 1q21. ${ }^{[17]}$ It has a molecular mass of $36.5 \mathrm{kDa}^{[18]}$ and is characterized by classical helix-loop-helix motif. ${ }^{[8]}$ Expression of S100A8 and S100A9 was found in granulocytes, monocytes and early differentiation states of macrophages. ${ }^{[10,19-21]}$ Edgeworth et al. ${ }^{[19]}$ figured out that S100A8 and S100A9 comprised approximately $46 \%$ of neutrophil cytosolic proteins. Moreover, murine microvascular endothelial cells, ${ }^{[2]}$ osteoblasts, osteoclasts, hypertrophic chondrocytes, and human bone marrow stromal stem cells ${ }^{[2,24]}$ were also reported to excrete the two proteins. S100A8 and S100A9 have utility in calcium management, cytoskeleton regulation and cell migration within cells. In the presence of calcium, S100A8/A9 complexes accelerate microtubule polymerization and tubulin filaments formation, accounting for cytoskeleton stabilization. ${ }^{[9]}$ The same study stated, in S100A9 ${ }^{--}$mice that lack functional S100A8/A9 complexes, tubulin in granulocytes decreased and infiltrating neutrophils in wound reduced, ${ }^{[9]}$ indicating the correlation of S100A8/A9 with cell migration. In addition, once secreted to extracellular space, CLP serves as DAMPs and binds to pattern recognition receptors (PRR), mediating cell differentiation, activation, migration, apoptosis, and production of pro-inflammatory factors. ${ }^{[10,11]}$ Evidence proves that CLP in monocyte is released actively via an unimpaired microtubule network following activation of protein kinase C. ${ }^{[25]}$ Over recent years, a report demonstrates that systemic and SF CLP levels prominently elevate in RA patients and the levels correlate positively with disease activity. ${ }^{[26]}$ Moreover, studies found aberrant CLP concentration in patients with inflammatory bowel disease (IBD), ankylosing spondylitis (AS), adult-onset Still's disease (AOSD) and systemic lupus erythematosus (SLE), ${ }^{[27-29]}$ revealing CLP may be relevant to the pathogenesis of multiple autoimmune diseases.

\section{TLR4 and related signaling pathway}

CLP is one of the endogenous ligands of TLR4, which belongs to PRRs family. TLR4 is a kind of type I membrane-spanning protein and is composed of an extracellular domain, a transmembrane domain, and intracellular domain. The ectodomain contains 22 leucinerich repeats (LRRs) mediating ligand recognition, while intracellular domain contains a Toll/IL-1 receptor (TIR) domain delivering downstream signal transduction. ${ }^{[30]}$

Some studies have demonstrated pro-inflammatory functions of CLP and TLR4 signaling pathway. TLR4 involves two distinct signaling pathways: myeloid differentiation primary response protein 88 (MyD88) dependent signal pathway on the membrane and MyD88-independent signal pathway in the endosomal compartment. ${ }^{[31]}$ We focus on the first pathway in this section. Once activated, two receptors form homodimer combined on the cell surface, leading to quick recruitment of adaptor proteins (such as MyD88, MyD88-adaptor-like protein also called as MAL or TIRAP), which contain TIR domains. ${ }^{[32]}$ Adaptor proteins produce downstream signals by phosphorylation of IL-1R-associated kinases (IRAKs), activation of TNF-receptor-associated factor 6 (TRAF6), and stimulation of transforming growth factor $\beta$-activated kinase 1 (TAK1). ${ }^{[32]}$ Subsequently, TAK1 activates the mitogen-activated protein kinases (MAPKs), including p38, JUN N-terminal kinase (JNK), extracellular signalregulated kinases (ERK1/2), and stimulates the IkB kinase complex (IKK), resulting in the regulation of transcription factors like NK- $x \mathrm{~B}$ and AP-1. ${ }^{[31-33]}$ However, few studies have been proposed to conclude other roles of the pathway in cell differentiation, migration and apoptosis.

TLR4 is detected on the membrane of phagocytes, endothelial cells, osteoclasts, chondrocytes, fibroblastlike synoviocytes (FLS). ${ }^{[34-38]}$ Selectively blocking CLP and TLR4 via the formation of (S100A8/A9), tetramers restricts the activity of these "alarmins" in vivo. ${ }^{[39]}$ Inversely, overactive TLR4 signaling pathways may aggravate inflammation and tissue damage. ${ }^{[16]}$ Another study clearly identified that S100A8 stimulates phagocytes activation and osteoclast differentiation by TLR4, respectively resulting in the inflammation and osteoclastic bone resorption ${ }^{[35,37]}$. Furthermore, S100A8/A9 activates p38 and NK- $x \mathrm{~B}$, and increases cytokines from macrophages of RA patients, including tumor necrosis factor- $\alpha$ (TNF- $\alpha$ ), interleukin (IL)$1 \beta$, IL- 6, IL- $8{ }^{[10]}$ As mentioned above, the TLR4 signaling pathway may play striking roles in the development of RA.

\section{THE ROLE OF CLP IN PATHOGENESIS OF RA}

RA is a common chronic arthritis typified by synovial hyperplasia, inflammatory cells infiltration in synovia, high expression of cytokines, and cartilage and bone erosion of joints. In RA, phagocytes including macrophages and neutrophils are abnormally activated and accumulated in the articular cavity, initiating multiple inflammatory factors (such as CLP) production and joint irreversible destruction. In fact, S100A8 and S100A9 are extensively expressed in 
synovium of experimental arthritis as compared with wildtype (W'T) mice. ${ }^{[40]}$ After adjuvant-induced arthritis (AIA) induction, joint swelling and bone erosion of WT mice were more serious than S100A9 ${ }^{-/}$mice. ${ }^{[35,40]}$ Therefore, S100A8/A9 may play momentous roles in the pathogenesis of RA.

CLP is mainly expressed by phagocytes. A study proved that monocytes interact with activated endothelial cells and secrete CLP. ${ }^{[41]}$ Interestingly, S100A8 is responsible for CLP activity whereas S100A9 protects S100A8 from degradation. ${ }^{[9,40]}$ The previous studies suggested TNF- $\alpha$, IFN- $\gamma$ and IL-10 promoted S100A8 mRNA expression in macrophages. ${ }^{[42,43]}$ In RA, the high levels of CLP may exert significant effects on distinct cells accumulated in joints. Firstly, CLP amplifies the production of inflammatory markers by phagocytes. Treated with $50 \mu \mathrm{g} / \mathrm{ml} \mathrm{CLP}$ (similar to RA SF CLP concentration), vitro-differentiated macrophages from healthy individuals were found to secrete TNF- $\alpha .{ }^{[10]}$ Similarly, CLP was proved to induce the production of TNF- $\alpha$, IL-1 $\beta$, IL- 6 , IL- 8 by purified blood monocytes. ${ }^{[10]}$ In an additional study, recombinant S100A8/A9 promoted the expression of MMP2, MMP3, MMP9 and MMP13 by murine macrophages. ${ }^{[40]}$ Secondly, CLP upregulated the CD11b/CD18 molecules on polymorphonuclear cells and monocytes, enhancing the combination of $\mathrm{CD} 11 \mathrm{~b}$ and intracellular adhesion molecule-1 (ICAM-1). ${ }^{[44,45]}$ By this way, CLP drove transendothelial migration of phagocytes and attracted them into the RA joints, leading to more CLP accumulated in the inflammatory site. Therefore, CLP may amplify the articular inflammation in RA patients.

CLP induces proteoglycans (PG) breakdown and chondrocytes apoptosis. PG loss and chondrocyte death were increased in the AIA models, while the deletion of CLP almost completely prevented cartilage destruction. ${ }^{[40]}$ Nitric oxide (NO) is an essential inflammatory mediator of RA, and it was observed to induce the apoptosis of the cultured articular chondrocytes. ${ }^{[1]}$ Interestingly, CLP can upregulate inducible nitric oxide synthase (iNOS) gene expression in macrophages, thus raising the NO concentration. ${ }^{[1]]}$ In addition, S100A8 (the active component of CLP) stimulated the activation of murine chondrocytes via NK- $x \mathrm{~B}$ pathway, subsequently promoting the release of inflammatory factors including IL-6, MMPs, and a disintegrin and metalloproteinase with thrombospondin motifs (ADAMTS). ${ }^{[23]}$ Consequently, CLP mediates cartilage destruction and participates in RA joint inflammation.

CLP also directly activates endothelial cells. It was reported that CLP induced ICAM-1 expression in human microvascular endothelial cells (HMECs), stimulated
HMECs to produce IL-8, which served as a chemoattractant for inflammatory cells, and increased vascular permeability. ${ }^{[46]}$ Moreover, another study also found an elevated permeability of human umbilical venous endothelial cells (HUVECs) monolayer via CLP in collaboration with TLR4 ${ }^{[47]}$ Based on the above results, CLP may play vital roles in endothelial cells and be responsible for inflammatory cell extravasation and recruitment during inflammation in RA.

CLP regulates osteoclast differentiation indirectly. As we know, upregulation of osteoclasts was related to bone erosion in joins. The previous study investigated that CLP had no impact on osteoclast formation during AIA, whereas S100A8 promoted osteoclast differentiation. ${ }^{[35]}$ Similarly, another research did not conclude the relationship between CLP and the number of osteoclasts in the joints of CIA. ${ }^{[48]}$ However, TNF- $\alpha$ and IL-6 levels induced by CLP enhanced osteoclasts differentiation in CIA. ${ }^{[48]}$

The accurate relevance of CLP and FLSs is not described. It is generally known that the activated FLSs secrete multiple inflammatory markers, perpetuating joint damage in RA. Studies proved that CLP levels raised in RA synovial tissue and TLR4 was highly expressed in FLSs. ${ }^{[34]} \mathrm{Up}$ to now, whether CLP exerts direct proinflammatory effects on FLSs have not been reported yet. However, treated with another TLR4 ligand (LPS), FLSs expressed higher IL-22, thus enhancing proliferation of FLSs, secretion of MMPs, S100A8, and S100A9. ${ }^{[49]}$ Accordingly, we suppose CLP has proinflammatory effects on FLSs and future well-designed investigations are needed.

\section{THE ROLE OF CLP IN CLINICAL ASSESSMENT OF RA}

CLP is a small molecule and released by activated macrophages, neutrophils, osteoblasts and other cells in arthritic joints. Evidence showed that blood and SF levels of CLP are significantly higher in the patients with RA as compared with controls. ${ }^{[2,50]}$ According to previous reports, CLP diffuses from the local joint cavity to circulation in RA and CLP SF levels are higher than serum. ${ }^{[2]}$ Serum CLP is easily measured and is considered to directly reflect the severity of arthritis. During the past decades, CLP levels have been reported to be connected with clinical and laboratory parameters, disease activity, synovitis progression determined by ultrasound (US) and radiology, and the therapeutic reaction of RA. CLP was more sensitive than traditional acute inflammatory markers (such as CRP) in the assessment of disease activity and US synovitis. ${ }^{[7,51]}$ Thus, CLP may be a potential inflammatory biomarker in RA. Next, we separately summarize the relationship between CLP concentrations and clinical signs, laboratory parameters, disease activity, 
ultrasound and radiographic progression of joints, and treatment response in RA.

Serum CLP levels are strongly correlated with clinical signs and acute phase reactants in RA. The noteworthy association between serum CLP and swollen joint count (SJC), tender joint count (TJC) was observed in several studies. ${ }^{[3,7,52,53]}$ CLP circulatory levels have significant correlations with grip strength $(r=-0.22, P<0.05)$ and circumferences of proximal interphalangeal (PIP) joints $(r=0.33, P<0.05)$ in a cross-sectional study of $70 \mathrm{RA}$ patients. ${ }^{[4]}$ Above studies also suggested an association of serum CLP and other serological indicators. ${ }^{[7,52,53]} \mathrm{A}$ systematic analysis highlighted that CLP was positively correlated with CRP and ESR $(r=0.8, P<0.01 ; r=0.7$, $P<0.01)$ in RA. ${ }^{[2]}$ In addition, serum CLP levels were also associated with rheumatoid factor (RF) levels $(r=0.25$, $P<0.05) .{ }^{[3]}$ The Rheumatoid Arthritis Articular Damage (RAAD) score serves as another clinical joint damage index. It was reported that CLP levels were positively relevant to RAAD score rather than CRP or ESR levels. ${ }^{[54]}$ Nevertheless, it was still contradictory whether CLP was related to anticitrullinated peptide antibodies titers. ${ }^{[3,50]}$

Association between serum CLP and disease activity of RA has been confirmed in recent years. Circulating CLP levels are high in active $\mathrm{RA}^{[2]}$ and are significantly related to Disease Activity Score based on a 28 -joint count (DAS28), simplified Disease Activity Index (SDAI) and Clinical Disease Activity Index (CDAI).$^{7,50,53,55]}$ The study yielded a CDAI score raised with elevated CLP level. ${ }^{[55]}$ In some RA patients, the levels of CRP or ESR were normal. Under this circumstance, CLP, prior to CRP, is a satisfactory predictor of CDAI activity in linear regression analyses. ${ }^{[7]}$ Hurnakova et al. ${ }^{[51]}$ found that the levels of CLP were lower in patients who were in remission or had low disease activity. Remarkably, all patients had normal or low CRP levels in this study. ${ }^{[51]}$ When RA patients who treated with tumor necrosis factor inhibitors ( $T N F i)$ were in remission, CLP levels were significantly lower. ${ }^{[5]}$ Thus, CLP is a good marker to assess the disease activity of RA patients receiving $\mathrm{TNFi}^{[56]}$

CLP may be essential to predict ultrasound and radiographic joints' progression of RA. US sum score is semi-quantitatively defined as the sum of Grey scale (GS) synovitis, power Doppler (PD) synovitis, GS tenosynovitis, and PD tenosynovitis. GS score is generally used for synovitis evaluation and PD score is for vascularization evaluation in the joints of RA. ${ }^{[52]}$ Studies demonstrated a correlation between CLP levels and US sum scores, GS and PD synovitis scores. ${ }^{[7,52]}$ Additionally, Hurnakova et al. ${ }^{[53]}$ analyzed the value of serum CLP levels for PD synovitis in RA by a multivariate regression analysis. When adjusted for age and sex, serum CLP levels $\left(R^{2}=0.765, P\right.$ $<0.001)$ were better than CRP $\left(R^{2}=0.496, P<0.001\right) .{ }^{[53]}$ RA patients in clinical remission are divided into two principal subgroups based on the musculoskeletal US: patients with US-defined remission and with subclinical disease activity. ${ }^{[5]}$ Interestingly, the CLP levels in the US-defined remission group were noticeably higher than those in the subclinical disease activity group (2.5 \pm 1.3 vs. $1.7 \pm 0.8 \mu \mathrm{g} / \mathrm{mL}, P<0.005),{ }^{[57]}$ indicating that CLP may have the utility to distinguish distinct groups of RA patients with clinical remission. Diverse studies concluded contradictory results about whether CLP was in connection with joint radiographic progression of RA. In a prospective single-center cohort study, 42 RA patients were assessed with serum CLP levels and dominant hand MRI at baseline and at 12 months. ${ }^{[1]}$ Baseline CLP level was not the independent predictor for MRI structural damage at 12 months. ${ }^{[1]}$ However, another group revealed high levels of CLP at baseline, but not CRP or ESR, were associated with joint radiographic progression. ${ }^{[55]}$ Similarly, Hammer et al. proved that CLP was independently correlated with joint radiographic damage in RA. ${ }^{[5]}$

Several groups have proved the relationship between CLP and treatment response in RA. 194 RA patients received methotrexate therapy were followed up for 4 months to determine if CLP has advantages in predicting the treatment response. Results showed that the circulating CLP levels at baseline can't predict treatment response. ${ }^{[55]}$ Nevertheless, other studies observed that the CLP levels in the patients with active RA decreased in treatment responders, while there was no remarkable change found in the non-responders. ${ }^{[2,3,54]}$

\section{CONCLUSION}

CLP participates in the pathological course of RA and acts as a biomarker. Deletion of CLP almost completely prevented cartilage destruction in the animal arthritis model. CLP exert their pro-inflammatory function via CLP/TLR4 signaling pathway. CLP can promote cytokine production from macrophages and endothelial cells, and regulate chondrocytes apoptosis. CLP can indirectly mediate osteoclasts differentiation. Furthermore, the levels of CLP are closely correlated with clinical signs, laboratory parameters, disease activity, ultrasound and radiographic progression, and the treatment response in RA patients. CLP is more useful than ESR and CRP to assess disease activity. In the future, more studies are required to validate the precise roles of CLP in the pathogenesis of RA and in the clinical assessment of RA patients. 


\section{Source of Foundation}

This study is supported in part by the grants from National Natural Science Foundation of China (81701600) and Natural Science Foundation of Zhejiang Province (LQ17H100001 and LGF18H100001).

\section{Conflict of Interests}

None declared.

\section{REFERENCE}

1. Ramirez J, Narvaez JA, Ruiz-Esquide V, Hernandez-Ganan J, Cuervo A, Inciarte-Mundo J, et al. Clinical and sonographic biomarkers of structural damage progression in RA patients in clinical remission: A prospective study with 12 months follow-up. Semin Arthritis Rheum 2017; 47:303-9.

2. Abildtrup M, Kingsley GH, Scott DL. Calprotectin as a biomarker for rheumatoid arthritis: a systematic review. J Rheumatol 2015; 42:760-70.

3. Garcia-Arias M, Pascual-Salcedo D, Ramiro S, Ueberschlag ME, Jermann TM, Cara C, et al. Calprotectin in rheumatoid arthritis : association with disease activity in a cross-sectional and a longitudinal cohort. Mol Diagn Ther 2013; 17:49-56

4. Brun JG, Haga HJ, Boe E, Kallay I, Lekven C, Berntzen HB, et al. Calprotectin in patients with rheumatoid arthritis: relation to clinical and laboratory variables of disease activity. J Rheumatol 1992; 19:859-62.

5. Brun JG, Jonsson R, Haga HJ. Measurement of plasma calprotectin as an indicator of arthritis and disease activity in patients with inflammatory rheumatic diseases. J Rheumatol 1994; 21:733-8.

6. Berntzen HB, Olmez U, Fagerhol MK, Munthe E. The leukocyte protein L1 in plasma and synovial fluid from patients with rheumatoid arthritis and osteoarthritis. Scand J Rheumatol 1991; 20:74-82.

7. Hurnakova J, Hulejova H, Zavada J, Hanova P, Komarc M, Mann H, et al. Relationship between serum calprotectin (S100A8/9) and clinical, laboratory and ultrasound parameters of disease activity in rheumatoid arthritis: A large cohort study. PLoS One 2017; 12:e0183420.

8. Ehrchen JM, Sunderkotter C, Foell D, Vogl T, Roth J. The endogenous Toll-like receptor 4 agonist S100A8/S100A9 (calprotectin) as innate amplifier of infection, autoimmunity, and cancer. J Leukoc Biol 2009; 86:557-66.

9. Vogl T, Ludwig S, Goebeler M, Strey A, Thorey IS, Reichelt R, et al. MRP8 and MRP14 control microtubule reorganization during transendothelial migration of phagocytes. Blood 2004; 104:4260-8.

10. Sunahori K, Yamamura M, Yamana J, Takasugi K, Kawashima M, Yamamoto $\mathrm{H}$, et al. The S100A8/A9 heterodimer amplifies proinflammatory cytokine production by macrophages via activation of nuclear factor kappa B and p38 mitogen-activated protein kinase in rheumatoid arthritis. Arthritis Res Ther 2006; 8:R69.

11. Malemud CJ. Myeloid-related protein activity in rheumatoid arthritis. Int J Inflam 2011; 2011:580295.

12. Moore BW. A soluble protein characteristic of the nervous system. Biochem Biophys Res Commun 1965; 19:739-44.

13. Donato R, Cannon BR, Sorci G, Riuzzi F, Hsu K, Weber DJ, et al. Functions of S100 proteins. Curr Mol Med 2013; 13:24-57.

14. Bertheloot D, Latz E. HMGB1, IL-1alpha, IL-33 and S100 proteins: dualfunction alarmins. Cell Mol Immunol 2017; 14:43-64.

15. Jiang $\mathrm{H}, \mathrm{Hu} \mathrm{H}$, Tong $\mathrm{X}$, Jiang Q, Zhu H, Zhang S. Calcium-binding protein $\mathrm{S} 100 \mathrm{P}$ and cancer: mechanisms and clinical relevance. J Cancer Res Clin Oncol 2012; 138:1-9.

16. O'Neill LA, Bryant CE, Doyle SL. Therapeutic targeting of Toll-like receptors for infectious and inflammatory diseases and cancer. Pharmacol Rev 2009; 61:177-197.
17. Marenholz I, Heizmann CW, Fritz G. S100 proteins in mouse and man: from evolution to function and pathology (including an update of the nomenclature). Biochem Biophys Res Commun 2004; 322:1111-22.

18. Dale I, Fagerhol MK, Naesgaard I. Purification and partial characterization of a highly immunogenic human leukocyte protein, the L1 antigen. Eur J Biochem 1983; 134:1-6.

19. Edgeworth J, Gorman M, Bennett R, Freemont P, Hogg N. Identification of p8,14 as a highly abundant heterodimeric calcium binding protein complex of myeloid cells. J Biol Chem 1991; 266:7706-13.

20. Dale I, Brandtzaeg P, Fagerhol MK, Scott H. Distribution of a new myelomonocytic antigen (L1) in human peripheral blood leukocytes. Immunofluorescence and immunoperoxidase staining features in comparison with lysozyme and lactoferrin. Am J Clin Pathol 1985; 84:24-34.

21. Di Ceglie I, Ascone G, Cremers NAJ, Sloetjes AW, Walgreen B, Vogl $\mathrm{T}$, et al. Fcgamma receptor-mediated influx of S100A8/A9-producing neutrophils as inducer of bone erosion during antigen-induced arthritis. Arthritis Res Ther 2018; 20:80.

22. Yen T, Harrison CA, Devery JM, Leong S, Iismaa SE, Yoshimura T, et al. Induction of the S100 chemotactic protein, CP-10, in murine microvascular endothelial cells by proinflammatory stimuli. Blood 1997; 90:4812-21.

23. van Lent PL, Grevers LC, Blom AB, Arntz OJ, van de Loo FA, van der Kraan $\mathrm{P}$, et al. Stimulation of chondrocyte-mediated cartilage destruction by S100A 8 in experimental murine arthritis. Arthritis Rheum 2008; 58:3776-87.

24. Zreiqat H, Howlett CR, Gronthos S, Hume D, Geczy CL. S100A8/ S100A9 and their association with cartilage and bone. J Mol Histol 2007; 38:381-91.

25. Rammes A, Roth J, Goebeler M, Klempt M, Hartmann M, Sorg C. Myeloid-related protein (MRP) 8 and MRP14, calcium-binding proteins of the $\$ 100$ family, are secreted by activated monocytes via a novel, tubulin-dependent pathway. J Biol Chem 1997; 272:9496-502.

26. Bae SC, Lee YH. Calprotectin levels in rheumatoid arthritis and their correlation with disease activity: a meta-analysis. Postgrad Med 2017; 129:531-7.

27. Klingberg E, Strid H, Stahl A, Deminger A, Carlsten H, Ohman L, et al. A longitudinal study of fecal calprotectin and the development of inflammatory bowel disease in ankylosing spondylitis. Arthritis Res Ther 2017; 19:21.

28. Guo Q, Zha X, Li C, Jia Y, Zhu L, Guo J, et al. Serum calprotectin--a promising diagnostic marker for adult-onset Still's disease. Clin Rheumatol 2016; 35:73-9.

29. Haga HJ, Brun JG, Berntzen HB, Cervera R, Khamashta M, Hughes GR. Calprotectin in patients with systemic lupus erythematosus: relation to clinical and laboratory parameters of disease activity. Lupus 1993; 2:47-50.

30. Akira S, Takeda K. Toll-like receptor signalling. Nat Rev Immunol 2004; 4:499-511.

31. Vallance TM, Zeuner MT, Williams HF, Widera D, Vaiyapuri S. Toll-Like Receptor 4 Signalling and Its Impact on Platelet Function, Thrombosis, and Haemostasis. Mediators Inflamm 2017; 2017:9605894.

32. Barton GM, Medzhitov R. Toll-like receptor signaling pathways. Science 2003; 300:1524-5.

33. Kawasaki T, Kawai T. Toll-like receptor signaling pathways. Front Immunol 2014; 5:461.

34. Ospelt C, Brentano F, Rengel Y, Stanczyk J, Kolling C, Tak PP, et al. Overexpression of toll-like receptors 3 and 4 in synovial tissue from patients with early rheumatoid arthritis: toll-like receptor expression in early and longstanding arthritis. Arthritis Rheum 2008; 58:3684-92.

35. Grevers LC, de Vries TJ, Vogl T, Abdollahi-Roodsaz S, Sloetjes AW, Leenen PJ, et al. S100A8 enhances osteoclastic bone resorption in vitro through activation of Toll-like receptor 4: implications for bone destruction in murine antigen-induced arthritis. Arthritis Rheum 2011; 63:1365-75. 
36. Molteni M, Gemma S, Rossetti C. The Role of Toll-Like Receptor 4 in Infectious and Noninfectious Inflammation. Mediators Inflamm 2016; 2016:6978936.

37. Fassl SK, Austermann J, Papantonopoulou O, Riemenschneider M, Xue $\mathrm{J}$, Bertheloot $\mathrm{D}$, et al. Transcriptome assessment reveals a dominant role for TLR4 in the activation of human monocytes by the alarmin MRP8. J Immunol 2015; 194:575-83.

38. Schelbergen RF, Blom AB, van den Bosch MH, Sloetjes A, AbdollahiRoodsaz S, Schreurs BW, et al. Alarmins S100A8 and S100A9 elicit a catabolic effect in human osteoarthritic chondrocytes that is dependent on Toll-like receptor 4. Arthritis Rheum 2012; 64:1477-87.

39. Vogl T, Stratis A, Wixler V, Voller T, Thurainayagam S, Jorch SK, et al. Autoinhibitory regulation of S100A8/S100A9 alarmin activity locally restricts sterile inflammation. J Clin Invest 2018; 128:1852-66.

40. van Lent PL, Grevers L, Blom AB, Sloetjes A, Mort JS, Vogl T, et al. Myeloid-related proteins S100A8/S100A9 regulate joint inflammation and cartilage destruction during antigen-induced arthritis. Ann Rheum Dis 2008; 67:1750-8.

41. De Rycke L, Baeten D, Foell D, Kruithof E, Veys EM, Roth J, et al. Differential expression and response to anti-TNFalpha treatment of infiltrating versus resident tissue macrophage subsets in autoimmune arthritis. J Pathol 2005; 206:17-27.

42. Xu K, Geczy CL. IFN-gamma and TNF regulate macrophage expression of the chemotactic S100 protein S100A8. J Immunol 2000; 164:4916-23.

43. Xu K, Yen T, Geczy CL. Il-10 up-regulates macrophage expression of the S100 protein S100A8. J Immunol 2001; 166:6358-66.

44. Eue I, Pietz B, Storck J, Klempt M, Sorg C. Transendothelial migration of 27E10+ human monocytes. Int Immunol 2000; 12:1593-604.

45. Newton RA, Hogg N. The human S100 protein MRP-14 is a novel activator of the beta 2 integrin Mac-1 on neutrophils. J Immunol 1998; 160:1427-35.

46. Viemann D, Strey A, Janning A, Jurk K, Klimmek K, Vogl T, et al. Myeloid-related proteins 8 and 14 induce a specific inflammatory response in human microvascular endothelial cells. Blood 2005; 105:2955-62.

47. Wang L, Luo H, Chen X, Jiang Y, Huang Q. Functional characterization of S100A8 and S100A9 in altering monolayer permeability of human umbilical endothelial cells. PLoS One 2014; 9:e90472.

48. Kiyeko GW, Hatterer E, Herren S, Di Ceglie I, van Lent PL, Reith W, et al. Spatiotemporal expression of endogenous TLR4 ligands leads to inflammation and bone erosion in mouse collagen-induced arthritis. Eur J Immunol 2016; 46:2629-38.
49. Carrion M, Juarranz Y, Martinez C, Gonzalez-Alvaro I, Pablos JL, Gutierrez-Canas I, et al. IL-22/IL-22R1 axis and S100A8/A9 alarmins in human osteoarthritic and rheumatoid arthritis synovial fibroblasts. Rheumatology (Oxford) 2013; 52:2177-86.

50. Mansour HE, Abdullrhman MA, Mobasher SA, El Mallah R, Abaza N, Hamed F, et al. Serum Calprotectin in Rheumatoid Arthritis: A Promising Diagnostic Marker, How Far Is It Related to Activity and Sonographic Findings? J Med Ultrasound 2017; 25:40-6.

51. Hurnakova J, Hulejova H, Zavada J, Komarc M, Cerezo LA, Mann H, et al. Serum calprotectin may reflect inflammatory activity in patients with active rheumatoid arthritis despite normal to low C-reactive protein. Clin Rheumatol 2018; 37:2055-62.

52. Nordal HH, Brokstad KA, Solheim M, Halse AK, Kvien TK, Hammer HB. Calprotectin (S100A8/A9) has the strongest association with ultrasound-detected synovitis and predicts response to biologic treatment: results from a longitudinal study of patients with established rheumatoid arthritis. Arthritis Res Ther 2017; 19:3.

53. Hurnakova J, Zavada J, Hanova P, Hulejova H, Klein M, Mann H, et al. Serum calprotectin (S100A8/9): an independent predictor of ultrasound synovitis in patients with rheumatoid arthritis. Arthritis Res Ther 2015; 17:252.

54. Hammer HB, Odegard S, Fagerhol MK, Landewe R, van der Heijde $\mathrm{D}$, Uhlig $\mathrm{T}$, et al. Calprotectin (a major leucocyte protein) is strongly and independently correlated with joint inflammation and damage in rheumatoid arthritis. Ann Rheum Dis 2007; 66:1093-7.

55. Jonsson MK, Sundlisaeter NP, Nordal HH, Hammer HB, Aga AB, Olsen IC, et al. Calprotectin as a marker of inflammation in patients with early rheumatoid arthritis. Ann Rheum Dis 2017; 76:2031-7.

56. Inciarte-Mundo J, Victoria Hernandez M, Ruiz-Esquide V, Raquel Cabrera-Villalba S, Ramirez J, Cuervo A, et al. Serum Calprotectin Versus Acute-Phase Reactants in the Discrimination of Inflammatory Disease Activity in Rheumatoid Arthritis Patients Receiving Tumor Necrosis Factor Inhibitors. Arthritis Care Res (Hoboken) 2016; 68:899-906.

57. Hurnakova J, Hulejova H, Zavada J, Komarc M, Hanova P, Klein M, et al. Serum Calprotectin Discriminates Subclinical Disease Activity from Ultrasound-Defined Remission in Patients with Rheumatoid Arthritis in Clinical Remission. PLoS One 2016; 11:e0165498.

How to cite this article: Wang Q, Chen W, Lin J. The Role of Calprotectin in Rheumatoid Arthritis. J Transl Int Med 2019; 7:126-31. 